Sādhanā Vol. 40, Part 1, February 2015, pp. 35-49. (C) Indian Academy of Sciences

\title{
New approach to solve fully fuzzy system of linear equations using single and double parametric form of fuzzy numbers
}

\author{
DIPTIRANJAN BEHERA and S CHAKRAVERTY*
}

\author{
Department of Mathematics, National Institute of Technology Rourkela, \\ Odisha 769 008, India \\ e-mail: diptiranjanb@gmail.com; sne_chak@yahoo.com
}

MS received 8 August 2012; revised 9 June 2014; accepted 2 September 2014

\begin{abstract}
This paper proposes two new methods to solve fully fuzzy system of linear equations. The fuzzy system has been converted to a crisp system of linear equations by using single and double parametric form of fuzzy numbers to obtain the non-negative solution. Double parametric form of fuzzy numbers is defined and applied for the first time in this paper for the present analysis. Using single parametric form, the $n \times n$ fully fuzzy system of linear equations have been converted to a $2 n \times 2 n$ crisp system of linear equations. On the other hand, double parametric form of fuzzy numbers converts the $n \times n$ fully fuzzy system of linear equations to a crisp system of same order. Triangular and trapezoidal convex normalized fuzzy sets are used for the present analysis. Known example problems are solved to illustrate the efficacy and reliability of the proposed methods.
\end{abstract}

Keywords. Fuzzy number; triangular fuzzy number; trapezoidal fuzzy number; parametric form of fuzzy number; fully fuzzy system of liner equations.

\section{Introduction}

System of linear equations has great applications in various areas such as operational research, physics, statistics, mathematics, engineering and social sciences. Equations of this type are necessary to solve the involved parameters. A general real system of linear equations may be written as $A X=b$, where, $A$ and $b$ are crisp real matrix and $X$ is unknown real vector. It is simple and straight forward when the variables involving the system of equations are crisp numbers. But in actual case the parameters may be uncertain or a vague estimation about the variables are known as those are found in general by some observation, experiment or experience. So, to overcome the uncertainty and vagueness, one may use the fuzzy numbers in place of the crisp numbers. Thus, the crisp system of linear equations becomes a Fuzzy System of Linear Equations (FSLE) or Fully Fuzzy System of Linear Equations (FFSLE). There is a difference between fuzzy linear system and fully fuzzy linear system. The coefficient matrix is treated as crisp in the fuzzy linear system, but in the fully fuzzy linear system all the parameters and variables are considered

*For correspondence 
to be fuzzy numbers. The concept of fuzzy set and fuzzy number were first introduced by Zadeh (1965). Related to fuzzy sets several excellent books have also been written by different authors (Hanss 2005; Zimmermann 2001; Ross 2004; Kaufmann \& Gupta 1988; Dubois \& Prade 1980). It is an important issue to develop mathematical models and numerical techniques that would appropriately treat the general fuzzy or fully fuzzy linear systems because subtraction and division of fuzzy numbers are not the inverse operations to addition and multiplication, respectively. So, this is an important area of research in the recent years. As such in the following paragraph few related literatures are reviewed for the sake of completeness of the problem.

Solution of a generalised $n \times n$ FSLE was first proposed by Friedman et al (1998), where the coefficient matrix and right-hand side column vector are defined as crisp and fuzzy, respectively. Some methods for solving this type of system can also be found in Allahviranloo (2004, 2005), Abbasbandy et al (2005), Sun \& Guo (2009), Yin \& Wang (2009), Chakraverty \& Behera (2013), Behera \& Chakraverty 2012, 2013e, Abbasbandy \& Jafarian (2006). Behera \& Chakraverty (2013a, c and d) applied this type of system to find the static responses of structures using fuzzy finite element method. Behera \& Chakraverty (2013b and 2014) have also presented various other methods to solve fuzzy complex system of linear equations.

On the other hand, FFSLE are also studied by various authors. Allahviranloo \& Mikaeilvand (2011a, b) discussed fully fuzzy system of linear equations using multi objective linear programming and the embedding approach. Recently, Das \& Chakraverty (2012) presented the numerical solution of interval and fuzzy system of linear equations analysing the circuit problems. Some iterative methods to solve fuzzy system of linear equations have been extended by Dehghan \& Hashemi (2006). Also Dehghan et al (2006, 2007) have proposed the adomian decomposition method, iterative methods and some computational methods such as Cramer's rule, Gauss elimination method, LU decomposition method and linear programming approach for finding the solutions of $n \times n$ fully fuzzy system of linear equations. Furthermore, Kumar et al (2011) have studied the fully fuzzy system of linear equations with an arbitrary fuzzy coefficient. A decomposition method was investigated by Mosleh et al (2009) to solve fully fuzzy system of linear equations. Muzzioli \& Reynaerts (2006) have considered fully fuzzy linear systems only in the form of $A_{1} x+b_{1}=A_{2} x+b_{2}$ with $A_{1}$ and $A_{2}$ are two square matrices of fuzzy numbers and $b_{1}$ and $b_{2}$ are fuzzy number vectors. Nasseri et al (2008) have used a certain decomposition method for the coefficient matrix to solve fully fuzzy linear systems. An excellent numerical approach based on Cholesky decomposition is described by Senthilkumar \& Rajendran (2011) to find positive solution of a symmetric fully fuzzy linear system. ST decomposition method for solving fully fuzzy linear systems using Gauss Jordan for trapezoidal fuzzy matrices is applied by Vijayalakshmi (2011). Recently, Babbar et al (2013) proposed a new method to find the nonnegative solution of a fully fuzzy linear system, where the elements of the coefficient matrix are defined as arbitrary triangular fuzzy numbers of the form $(m, \alpha, \beta)$. Muzzioli \& Reynaerts (2007) have investigated the non-negative solution procedure of fuzzy system by nonlinear programming approach. Otadi \& Mosleh (2012) have applied a linear programming approach to find the non-negative solution of a fully fuzzy matrix equation whose elements of the coefficient matrix are considered as arbitrary triangular fuzzy numbers. There are no restrictions about the elements of the coefficient matrix of the corresponding system.

In this paper, we have obtained a non-negative solution of a fully fuzzy linear system viz. $[\tilde{A}]\{\tilde{X}\}=\{\tilde{b}\}$, where $\tilde{A}, \tilde{X}$ and $\tilde{b} \geq 0$. The coefficient matrix $[\tilde{A}]=\left(\tilde{a}_{k j}\right), 1 \leq k, j \leq n$ is a fuzzy $n \times n$ matrix, $\{\tilde{b}\}=\left\{\tilde{b}_{k}\right\}, 1 \leq k$ is a column vector of fuzzy numbers and $\{\tilde{X}\}=\left\{\tilde{x}_{j}\right\}$ is the vector of fuzzy unknowns.

The present paper is organized as follows. In section 2, we have given some basic preliminaries related to the present investigation. The concepts of these have been used for the solution of fully 
fuzzy system of linear equation. We proposed two new methods to solve fully fuzzy system of linear equations in section 3 by using the single and double parametric form of fuzzy numbers. In section 4, numerical examples are solved using the proposed methods and are compared with the existing results. Finally, conclusions are provided in section 5.

\section{Preliminaries}

In the following paragraph some definitions related to the present work are given (Hanss 2005; Dubois \& Prade 1980; Zadeh et al 1975; Zimmermann 2001).

\subsection{Definition: Fuzzy number}

A fuzzy number $\tilde{U}$ is convex normalized fuzzy set $\tilde{U}$ of the real line $R$ such that

$$
\left\{\mu_{\tilde{U}}(x): R \rightarrow[0,1], \forall x \in R\right\},
$$

where $\mu_{\tilde{U}}$ is called the membership function of the fuzzy set and it is piece-wise continuous.

\subsection{Definition: Triangular fuzzy number}

A triangular fuzzy number $\tilde{U}$ is a convex normalized fuzzy set $\tilde{U}$ of the real line $R$ such that

(i) There exists exactly one $x_{0} \in R$ with $\mu_{\tilde{U}}\left(x_{0}\right)=1\left(x_{0}\right.$ is called the mean value of $\left.\tilde{U}\right)$, where $\mu_{\tilde{U}}$ is called the membership function of the fuzzy set.

(ii) $\mu_{\tilde{U}}(x)$ is piece-wise continuous.

Let us consider an arbitrary triangular fuzzy number $\tilde{U}=(a, b, c)$ as shown in figure 1a. The membership function $\mu_{\tilde{U}}$ of $\tilde{U}$ will be define as follows

$$
\mu_{\tilde{U}}(x)=\left\{\begin{array}{ll}
0, & x \leq a \\
\frac{x-a}{b-a}, & a \leq x \leq b \\
\frac{c-x}{c-b}, & b \leq x \leq c \\
0, & x \geq c
\end{array} .\right.
$$

\subsection{Definition: Trapezoidal fuzzy number}

Now, let us consider a trapezoidal fuzzy number as $\tilde{U}=(a, b, c, d)$ as shown in figure $1 \mathrm{~b}$. The membership function $\mu_{\tilde{U}}$ of $\tilde{U}$ will be interpreted as follows

$$
\mu_{\tilde{U}}(x)=\left\{\begin{array}{ll}
0, & x \leq a \\
\frac{x-a}{b-a}, & a \leq x \leq b \\
1, & b \leq x \leq c \\
\frac{d-x}{d-c}, & c \leq x \leq d \\
0, & x \geq d
\end{array} .\right.
$$



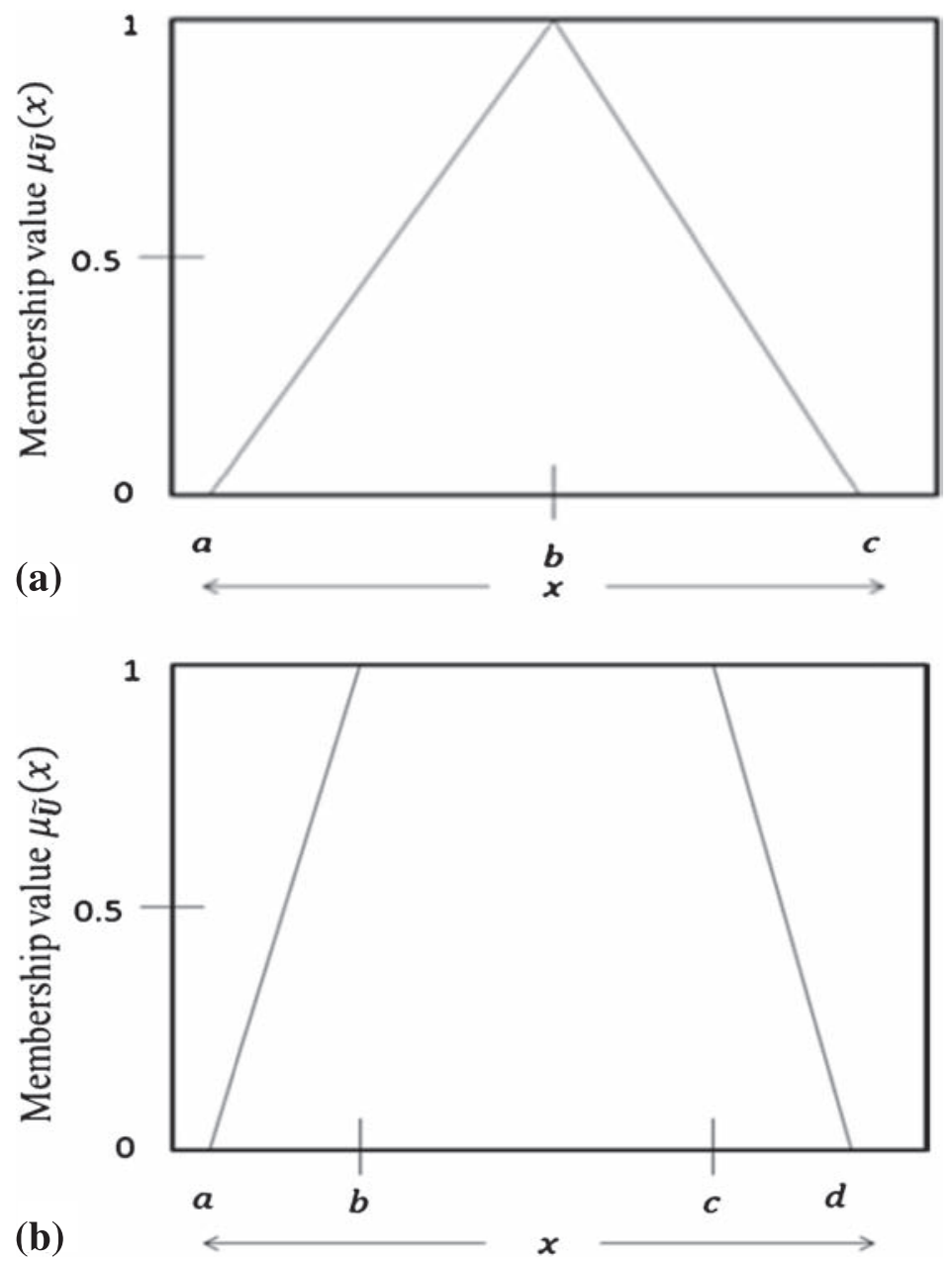

Figure 1. (a) Triangular fuzzy number. (b) Trapezoidal fuzzy number.

\subsection{Definition: Single parametric form of fuzzy numbers}

The triangular fuzzy number $\tilde{U}=(a, b, c)$ can be represented with an ordered pair of functions through $\alpha$-cut approach as $[\underline{u}(\alpha), \bar{u}(\alpha)]=[(b-a) \alpha+a,-(c-b) \alpha+c]$, where $\alpha \in[0,1]$. Also the trapezoidal fuzzy number $\tilde{U}=(a, b, c, d)$ can be represented with an ordered pair of functions through $\alpha$-cut approach as $[\underline{u}(\alpha), \bar{u}(\alpha)]=[(b-a) \alpha+a,-(d-c) \alpha+d]$, where $\alpha \in[0,1]$. The $\alpha$-cut form is known as parametric form or single parametric form of fuzzy numbers.

It may be noted that the lower and upper bounds of the fuzzy numbers satisfies the following requirements.

(i) $\underline{u}(\alpha)$ is a bounded left continuous non-decreasing function over $[0,1]$

(ii) $\overline{\bar{u}}(\alpha)$ is a bounded right continuous non-increasing function over $[0,1]$.

(iii) $\underline{u}(\alpha) \leq \bar{u}(\alpha), 0 \leq \alpha \leq 1$. 


\subsection{Definition: Double parametric form of fuzzy number}

Using the parametric form as defined in definition 2.3 we have $\tilde{U}=[\underline{u}(\alpha), \bar{u}(\alpha)]$. Now, one may represent this in crisp number using double parametric form as $\tilde{U}(\alpha, \beta)=\beta(\bar{u}(\alpha)-\underline{u}(\alpha))+\underline{u}(\alpha)$ where $\alpha$ and $\beta \in[0,1]$.

\subsection{Definition: Positive fuzzy number}

A fuzzy number $\tilde{U}$ is said to be positive, denoted by $\tilde{U}>0$ if its membership function $\mu_{\tilde{U}}(x)$ satisfies $\mu_{\tilde{U}}(x)=0, \forall x \leq 0$.

\subsection{Definition: Non-negative fuzzy number}

A fuzzy number $\tilde{U}$ is said to be non-negative, denoted by $\tilde{U} \geq 0$ if its membership function $\mu_{\tilde{U}}(x)$ satisfies $\mu_{\tilde{U}}(x)=0, \forall x<0$.

\subsection{Definition: Fuzzy matrix}

A matrix $[\tilde{A}]=\left(\tilde{a}_{k j}\right)$ is called a fuzzy matrix, if each element of $[\tilde{A}]$ is a fuzzy number. A fuzzy matrix $[\tilde{A}]$ will be non-negative, denoted by $[\tilde{A}] \geq 0$, if each element of $[\tilde{A}]$ will be a non-negative fuzzy number.

\subsection{Definition: Fuzzy arithmetic}

As discussed above, fuzzy numbers may be transformed into an interval through parametric form. So, for any arbitrary fuzzy number $\tilde{x}=[\underline{x}(\alpha), \bar{x}(\alpha)], \tilde{y}=[y(\alpha), \tilde{y}(\alpha)]$ and scalar $k$, we have the interval based fuzzy arithmetic as

(i) $\tilde{x}=\tilde{y}$ if and only if $\underline{x}(\alpha)=y(\alpha)$ and $\bar{x}(\alpha)=\bar{y}(\alpha)$,

(ii) $\tilde{x}+\tilde{y}=[\underline{x}(\alpha)+y(\alpha), \bar{x}(\alpha)+\bar{y}(\alpha)]$

(iii) $\tilde{x}-\tilde{y}=[\underline{x}(\alpha)-\overline{\bar{y}}(\alpha), \bar{x}(\alpha)-\underline{y}(\alpha)]$

(iv) $\tilde{x} \times \tilde{y}=[\min (S), \max (S)]$, where $S=\{\underline{x}(\alpha) \times \underline{y}(\alpha), \underline{x}(\alpha) \times \bar{y}(\alpha), \bar{x}(\alpha) \times \underline{y}(\alpha), \bar{x}(\alpha) \times \bar{y}(\alpha)\}$

(v) $k \tilde{x}= \begin{cases}{[k \bar{x}(\alpha), k \underline{x}(\alpha)],} & k<0 \\ {[k \underline{x}(\alpha), k \bar{x}(\alpha)],} & k \geq 0\end{cases}$

\section{Fully fuzzy system of linear equations and the proposed methods}

The $n \times n$ fully fuzzy system of linear equations may be written as

$$
\begin{gathered}
\tilde{a}_{11} \tilde{x}_{1}+\tilde{a}_{12} \tilde{x}_{2}+\cdots+\tilde{a}_{1 n} \tilde{x}_{n}=\tilde{b}_{1}, \\
\tilde{a}_{21} \tilde{x}_{1}+\tilde{a}_{22} \tilde{x}_{2}+\cdots+\tilde{a}_{2 n} \tilde{x}_{n}=\tilde{b}_{2}, \\
\vdots \\
\tilde{a}_{n 1} \tilde{x}_{1}+\tilde{a}_{n 2} \tilde{x}_{2}+\cdots+\tilde{a}_{n n} \tilde{x}_{n}=\tilde{b}_{n} .
\end{gathered}
$$

In matrix notation the above system may be written as $[\tilde{A}]\{\tilde{X}\}=\{\tilde{b}\}$, where the coefficient matrix $[\tilde{A}]=\left(\tilde{a}_{k j}\right), 1 \leq k, j \leq n$ is a fuzzy $n \times n$ matrix, $\{\tilde{b}\}=\left\{\tilde{b}_{k}\right\}, 1 \leq k$ is a column vector of fuzzy numbers and $\{\tilde{X}\}=\left\{\tilde{x}_{j}\right\}$ is the vector of fuzzy unknowns. Here we have assumed $\tilde{A}, \tilde{b}$ and $\tilde{X} \geq 0$. 


\subsection{Limitations of the existing methods}

In this subsection, we have pointed out some short comings of the existing methods to solve the considered fully fuzzy system of linear equations.

(i) Das \& Chakraverty (2012) studied the solution of $n \times n$ fully fuzzy system of linear equations by converting it into a $2 n \times 2 n$ crisp system of linear equations. The matrices involved in the corresponding system are considered as positive.

(ii) Cholesky decomposition was adopted by Senthilkumar \& Rajendran (2011) for the solution of a symmetric fully fuzzy system of linear equations. Here, positive matrices are considered and the elements are assumed as triangular fuzzy number. In this method, symmetric coefficient matrix has been decomposed into two matrices and then the solution was obtained in three steps.

(iii) Fully fuzzy linear systems can be solved by linear programming approach, Gauss elimination method, Cramer's rule, etc. (Dehghan et al 2006, 2007). These computational methods have various disadvantages like number of iterations, triangularisation, finding more number of determinants, etc.

To overcome these drawbacks, we have introduced new methods for solving fully fuzzy linear systems based on single and double parametric form of fuzzy numbers.

\subsection{Solution method using single parametric form}

The above system, $[\tilde{A}]\{\tilde{X}\}=\{\tilde{b}\}$ can be represented as

$$
\sum_{j=1}^{n} \tilde{a}_{k j} \tilde{x}_{j}=\tilde{b}_{k} \quad \text { for } k=1,2, \cdots, n \text {. }
$$

Using the parametric form of fuzzy number we may write the elements of the fuzzy coefficient matrix, real fuzzy unknown and the right hand real fuzzy number vector as $\tilde{a}_{k j}=$ $\left[\underline{a}_{k j}(\alpha), \bar{a}_{k j}(\alpha)\right], \tilde{x}_{j}=\left[\underline{x}_{j}(\alpha), \bar{x}_{j}(\alpha)\right]$ and $\tilde{b}_{k}=\left[\underline{b}_{k}(\alpha), \bar{b}_{k}(\alpha)\right]$.

Substituting the above expressions in Eq. (2), one may obtain the following equation as

$$
\sum_{j=1}^{n}\left[\underline{a}_{k j}(\alpha), \bar{a}_{k j}(\alpha)\right]\left[\underline{x}_{j}(\alpha), \bar{x}_{j}(\alpha)\right]=\left[\underline{b}_{k}(\alpha), \bar{b}_{k}(\alpha)\right] \text { for } k=1,2, \cdots, n .
$$

Eq. (3) can equivalently be expressed as the following two crisp Eqs. (4) and (5) by applying standard rule of fuzzy arithmetic as

$$
\sum_{j=1}^{n} \underline{a}_{k j}(\alpha) \underline{x}_{j}(\alpha)=\underline{b}_{k}(\alpha)
$$

and

$$
\sum_{j=1}^{n} \bar{a}_{k j}(\alpha) \bar{x}_{j}(\alpha)=\bar{b}_{k}(\alpha)
$$

One may write explicitly the combined form of Eqs. (4) and (5) as

$$
\left(\begin{array}{ll}
S & O \\
O & D
\end{array}\right)\left(\begin{array}{l}
y \\
z
\end{array}\right)=\left(\begin{array}{l}
p \\
q
\end{array}\right)
$$


where

$$
\begin{aligned}
& S=\left(\begin{array}{cccc}
\underline{a}_{11}(\alpha) & \underline{a}_{12}(\alpha) & \cdots & \underline{a}_{1 n}(\alpha) \\
\underline{a}_{21}(\alpha) & \underline{a}_{22}(\alpha) & \cdots & \underline{a}_{2 n}(\alpha) \\
\vdots & \vdots & \cdots & \vdots \\
\underline{a}_{n 1}(\alpha) & \underline{a}_{n 2}(\alpha) & \cdots & \underline{a}_{n n}(\alpha)
\end{array}\right), \quad D=\left(\begin{array}{cccc}
\bar{a}_{11}(\alpha) & \bar{a}_{12}(\alpha) & \cdots & \bar{a}_{1 n}(\alpha) \\
\bar{a}_{21}(\alpha) & \bar{a}_{22}(\alpha) & \cdots & \bar{a}_{2 n}(\alpha) \\
\vdots & \vdots & \cdots & \vdots \\
\bar{a}_{n 1}(\alpha) & \bar{a}_{n 2}(\alpha) & \cdots & \bar{a}_{n n}(\alpha)
\end{array}\right) \\
& y=\left(\begin{array}{c}
\underline{x}_{1}(\alpha) \\
\underline{x}_{2}(\alpha) \\
\vdots \\
\underline{x}_{n}(\alpha)
\end{array}\right), \quad z=\left(\begin{array}{c}
\bar{x}_{1}(\alpha) \\
\bar{x}_{2}(\alpha) \\
\vdots \\
\bar{x}_{n}(\alpha)
\end{array}\right), \quad p=\left(\begin{array}{c}
\underline{b}_{1}(\alpha) \\
\underline{b}_{2}(\alpha) \\
\vdots \\
\underline{b}_{n}(\alpha)
\end{array}\right), \quad q=\left(\begin{array}{c}
\bar{b}_{1}(\alpha) \\
\bar{b}_{2}(\alpha) \\
\vdots \\
\bar{b}_{n}(\alpha)
\end{array}\right)
\end{aligned}
$$

and $O$ represents $n \times n$ zero matrix.

Now, one may solve either Eqs. (4) and (5) separately or Eq. (6) directly to obtain the lower and upper bounds of the solution vector.

One may note that the procedure converts the fuzzy system to crisp system for the solution. Here, we have to solve either two crisp system separately or a single $2 n \times 2 n$ system. Hence to reduce the computational cost, a new approach is proposed in the following subsection based on double parametric form of fuzzy numbers.

\subsection{Solution method using double parametric form}

From the above discussion, one may represent system (1) in single parametric form as

$$
\sum_{j=1}^{n}\left[\underline{a}_{k j}(\alpha), \bar{a}_{k j}(\alpha)\right]\left[\underline{x}_{j}(\alpha), \bar{x}_{j}(\alpha)\right]=\left[\underline{b}_{k}(\alpha), \bar{b}_{k}(\alpha)\right] \text { for } k=1,2, \cdots, n \text {. }
$$

Next, using the double parametric form (as discussed in Definition 2.5), the elements of the fuzzy coefficient matrix, fuzzy unknown vector and right hand side fuzzy number vector of the above system can be expressed respectively as

$$
\begin{gathered}
{\left[\underline{a}_{k j}(\alpha), \bar{a}_{k j}(\alpha)\right]=\beta\left(\bar{a}_{k j}(\alpha)-\underline{a}_{k j}(\alpha)\right)+\underline{a}_{k j}(\alpha),} \\
{\left[\underline{x}_{j}(\alpha), \bar{x}_{j}(\alpha)\right]=\beta\left(\bar{x}_{j}(\alpha)-\underline{x}_{j}(\alpha)\right)+\underline{x}_{j}(\alpha) \text { and }} \\
{\left[\underline{b}_{k}(\alpha), \bar{b}_{k}(\alpha)\right]=\beta\left(\bar{b}_{k}(\alpha)-\underline{b}_{k}(\alpha)\right)+\underline{b}_{k}(\alpha) .}
\end{gathered}
$$

Substituting these expressions in the above system we may have

$$
\begin{aligned}
& \sum_{j=1}^{n}\left\{\beta\left(\bar{a}_{k j}(\alpha)-\underline{a}_{k j}(\alpha)\right)+\underline{a}_{k j}(\alpha)\right\}\left\{\beta\left(\bar{x}_{j}(\alpha)-\underline{x}_{j}(\alpha)\right)+\underline{x}_{j}(\alpha)\right\} \\
& =\beta\left(\bar{b}_{k}(\alpha)-\underline{b}_{k}(\alpha)\right)+\underline{b}_{k}(\alpha) .
\end{aligned}
$$

Let us define $\beta\left(\bar{x}_{j}(\alpha)-\underline{x}_{j}(\alpha)\right)+\underline{x}_{j}(\alpha)=\tilde{x}_{j}(\alpha, \beta)$ and then we substitute this in Eq. (7) to get

$$
\sum_{j=1}^{n}\left\{\beta\left(\bar{a}_{k j}(\alpha)-\underline{a}_{k j}(\alpha)\right)+\underline{a}_{k j}(\alpha)\right\}\left\{\tilde{x}_{j}(\alpha, \beta)\right\}=\beta\left(\bar{b}_{k}(\alpha)-\underline{b}_{k}(\alpha)\right)+\underline{b}_{k}(\alpha) .
$$


The above Eq. (8) is now symbolically solved to obtain $\tilde{x}_{j}(\alpha, \beta)$. After getting the expression of $\tilde{x}_{j}(\alpha, \beta)$ one may substitute $\beta=0$ and 1 to get the lower and upper bounds of the fuzzy solution vector, respectively. Accordingly this gives $\tilde{x}_{j}(\alpha, 0)=\underline{x}_{j}(\alpha)$ and $\tilde{x}_{j}(\alpha, 1)=\bar{x}_{j}(\alpha)$.

The order of the main system remains unaltered in this solution procedure. So the method is computationally efficient in comparison with other methods. Also the method is straight forward and easy to handle because the fuzzy system turns in to a crisp system using double parametric form of fuzzy numbers.

\subsection{Existence of a suitable solution}

In this subsection a theorem is stated and proved as follows for the existence of solution. One may express the non-negative system (1) as

$$
[\tilde{A}(\alpha, \beta)]\{\tilde{X}(\alpha, \beta)\}=\{\tilde{b}(\alpha, \beta)\},
$$

using the double parametric form of the fuzzy number.

Theorem 3.1 Let $\tilde{A}(\alpha, \beta) \geq 0, \tilde{b}(\alpha, \beta) \geq 0$ and $\tilde{A}(\alpha, \beta)$ correspond to a permutation matrix. Then the non-negative fully fuzzy system of linear equations has a non-negative consistent fuzzy solution.

Proof. Hypotheses imply that $[\tilde{A}(\alpha, \beta)]^{-1}$ exists as non-negative matrix (DeMarr 1972). So we have $\{\tilde{X}(\alpha, \beta)\}=[\tilde{A}(\alpha, \beta)]^{-1}\{\tilde{b}(\alpha, \beta)\} \geq 0$. Hence one may conclude that $\{\tilde{X}(\alpha, \beta)\}$ is a non-negative solution of the required system.

\section{Numerical examples}

To illustrate the applicability of the proposed method, two numerical example problems and two real world application problems are solved in this section.

Example 1. Let us consider a $2 \times 2$ fully fuzzy system of linear equations (Allahviranloo \& Mikaeilvand 2011a; Dehghan et al 2006)

$$
\begin{gathered}
{[4+\alpha, 6-\alpha] \tilde{x}_{1}+[5+\alpha, 8-2 \alpha] \tilde{x}_{2}=[40+10 \alpha, 67-17 \alpha],} \\
{[6+\alpha, 7] \tilde{x}_{1}+[4,5-\alpha] \tilde{x}_{2}=[43+5 \alpha, 55-7 \alpha] .}
\end{gathered}
$$

\subsection{Solution using single parametric form}

By Eqs. (4) and (5) this can be written as

$$
\begin{gathered}
(4+\alpha) \underline{x}_{1}(\alpha)+(5+\alpha) \underline{x}_{2}(\alpha)=40+10 \alpha, \\
(6+\alpha) \underline{x}_{1}(\alpha)+4 \underline{x}_{2}(\alpha)=43+5 \alpha, \\
(6-\alpha) \bar{x}_{1}(\alpha)+(8-2 \alpha) \bar{x}_{2}(\alpha)=67-17 \alpha, \\
7 \bar{x}_{1}(\alpha)+(5-\alpha) \bar{x}_{2}(\alpha)=55-7 \alpha .
\end{gathered}
$$


In matrix notation the above system can be written as

$$
\left(\begin{array}{cccc}
4+\alpha & 5+\alpha & 0 & 0 \\
6+\alpha & 4 & 0 & 0 \\
0 & 0 & 6-\alpha & 8-2 \alpha \\
0 & 0 & 7 & 5-\alpha
\end{array}\right)\left(\begin{array}{c}
x_{1}(\alpha) \\
\underline{x}_{2}(\alpha) \\
\bar{x}_{1}(\alpha) \\
\bar{x}_{2}(\alpha)
\end{array}\right)=\left(\begin{array}{c}
40+10 \alpha \\
43+5 \alpha \\
67-17 \alpha \\
55-7 \alpha
\end{array}\right)
$$

Now solving the above system of linear equations one may have

$$
\begin{gathered}
\underline{x}_{1}(\alpha)=\frac{5 \alpha^{2}+28 \alpha+55}{\alpha^{2}+7 \alpha+14}, \\
\underline{x}_{2}(\alpha)=\frac{5 \alpha^{2}+37 \alpha+68}{\alpha^{2}+7 \alpha+14}, \\
\bar{x}_{1}(\alpha)=\frac{3 \alpha^{2}+14 \alpha-105}{\alpha^{2}+3 \alpha-26} \text { and } \\
\bar{x}_{2}(\alpha)=\frac{7 \alpha^{2}+22 \alpha-139}{\alpha^{2}+3 \alpha-26} .
\end{gathered}
$$

Then we get the elements of the fuzzy solution vector as

$$
\tilde{x}_{1}=\left[\underline{x}_{1}(\alpha), \bar{x}_{1}(\alpha)\right]=\left[\frac{5 \alpha^{2}+28 \alpha+55}{\alpha^{2}+7 \alpha+14}, \frac{3 \alpha^{2}+14 \alpha-105}{\alpha^{2}+3 \alpha-26}\right] \text {, }
$$

and

$$
\tilde{x}_{2}=\left[\underline{x}_{2}(\alpha), x_{2}(\alpha)\right]=\left[\frac{5 \alpha^{2}+37 \alpha+68}{\alpha^{2}+7 \alpha+14}, \frac{7 \alpha^{2}+22 \alpha-139}{\alpha^{2}+3 \alpha-26}\right] .
$$

\subsection{Solution using double parametric form}

The original system can be represented by using the double parametric form of fuzzy numbers as

$$
\begin{aligned}
& \{\beta((6-\alpha)-(4+\alpha))+(4+\alpha)\}\left\{\beta\left(\bar{x}_{1}(\alpha)-\underline{x}_{1}(\alpha)\right)+\underline{x}_{1}(\alpha)\right\} \\
& +\{\beta((8-2 \alpha)-(5+\alpha))+(5+\alpha)\}\left\{\beta\left(\bar{x}_{2}(\alpha)-\underline{x}_{2}(\alpha)\right)+\underline{x}_{2}(\alpha)\right\} \\
& =\beta((67-17 \alpha)-(40+10 \alpha))+(40+10 \alpha), \\
& \quad\{\beta(7-(6+\alpha))+(6+\alpha)\}\left\{\beta\left(\bar{x}_{1}(\alpha)-\underline{x}_{1}(\alpha)\right)+\underline{x}_{1}(\alpha)\right\} \\
& \quad+\{\beta((5-\alpha)-4)+4\}\left\{\beta\left(\bar{x}_{2}(\alpha)-\underline{x}_{2}(\alpha)\right)+\underline{x}_{2}(\alpha)\right\} \\
& \quad=\beta((55-7 \alpha)-(43+5 \alpha))+(43+5 \alpha) .
\end{aligned}
$$

Let us consider $\beta\left(\bar{x}_{j}(\alpha)-\underline{x}_{j}(\alpha)\right)+\underline{x}_{j}(\alpha)=\tilde{x}_{j}(\alpha, \beta)$ for $j=1,2$. So substituting this value in the above system, it can be represented as

$$
\begin{aligned}
& \{\beta((6-\alpha)-(4+\alpha))+(4+\alpha)\} \tilde{x}_{1}(\alpha, \beta)+\{\beta((8-2 \alpha)-(5+\alpha))+(5+\alpha)\} \tilde{x}_{2}(\alpha, \beta) \\
& =\beta((67-17 \alpha)-(40+10 \alpha))+(40+10 \alpha), \\
& \quad\{\beta(7-(6+\alpha))+(6+\alpha)\} \tilde{x}_{1}(\alpha, \beta)+\{\beta((5-\alpha)-4)+4\} \tilde{x}_{2}(\alpha, \beta) \\
& \quad=\beta((55-7 \alpha)-(43+5 \alpha))+(43+5 \alpha) .
\end{aligned}
$$


Solving the above one may get

$$
\begin{aligned}
& \tilde{x}_{1}(\alpha, \beta)=\frac{9 \alpha^{2} \beta^{2}-17 \alpha^{2} \beta+5 \alpha^{2}-18 \alpha \beta^{2}-24 \alpha \beta+28 \alpha+9 \beta^{2}+41 \beta+55}{\alpha^{2} \beta^{2}-3 \alpha^{2} \beta+\alpha^{2}-2 \alpha \beta^{2}-8 \alpha \beta+7 \alpha+\beta^{2}+11 \beta+14}, \\
& \tilde{x}_{2}(\alpha, \beta)=\frac{3 \alpha^{2} \beta^{2}-15 \alpha^{2} \beta+5 \alpha^{2}-6 \alpha \beta^{2}-53 \alpha \beta+37 \alpha+3 \beta^{2}+68 \beta+68}{\alpha^{2} \beta^{2}-3 \alpha^{2} \beta+\alpha^{2}-2 \alpha \beta^{2}-8 \alpha \beta+7 \alpha+\beta^{2}+11 \beta+14} .
\end{aligned}
$$

Substituting $\beta=0$ and 1 in $\tilde{x}_{1}(\alpha, \beta)$ one may get the lower and upper bounds of the fuzzy solution respectively as

$$
\begin{gathered}
\tilde{x}_{1}(\alpha, 0)=\underline{x}_{1}(\alpha)=\frac{5 \alpha^{2}+28 \alpha+55}{\alpha^{2}+7 \alpha+14} \text { and } \\
\tilde{x}_{1}(\alpha, 1)=\bar{x}_{1}(\alpha)=\frac{3 \alpha^{2}+14 \alpha-105}{\alpha^{2}+3 \alpha-26} .
\end{gathered}
$$

Similarly substituting $\beta=0$ and 1 in $\tilde{x}_{2}(\alpha, \beta)$ we have

$$
\begin{gathered}
\tilde{x}_{2}(\alpha, 0)=\underline{x}_{2}(\alpha)=\frac{5 \alpha^{2}+37 \alpha+68}{\alpha^{2}+7 \alpha+14} \text { and } \\
\tilde{x}_{2}(\alpha, 1)=\bar{x}_{2}(\alpha)=\frac{7 \alpha^{2}+22 \alpha-139}{\alpha^{2}+3 \alpha-26} .
\end{gathered}
$$

Allahviranloo \& Mikaeilvand (2011a) and Dehghan et al (2006) have also solved this problem. Hence results obtained by the proposed methods are compared with the results of Allahviranloo and Mikaeilvand (2011a) and Dehghan et al (2006) and are shown in table 1.

It is interesting to note that method of Dehghan et al (2006) gives the approximate solution, wherever proposed methods give exact solution. Also one may notice that the results obtained by the present methods are exactly same as Allahviranloo \& Mikaeilvand (2011a).

Table 1. Comparison between Allahviranloo \& Mikaeilvand (2011a), Dehghan et al (2006) and present method(s).

\begin{tabular}{lccc}
\hline Solution bounds & Allahviranloo \& Mikaeilvand (2011a) & Dehghan et al (2006) & Present method(s) \\
\hline$\underline{x}_{1}(\alpha)$ & $\frac{5 \alpha^{2}+28 \alpha+55}{\alpha^{2}+7 \alpha+14}$ & $\frac{43}{11}+\frac{\alpha}{11}$ & $\frac{5 \alpha^{2}+28 \alpha+55}{\alpha^{2}+7 \alpha+14}$ \\
$\bar{x}_{1}(\alpha)$ & $\frac{3 \alpha^{2}+14 \alpha-105}{\alpha^{2}+3 \alpha-26}$ & 4 & $\frac{3 \alpha^{2}+14 \alpha-105}{\alpha^{2}+3 \alpha-26}$ \\
$\underline{x}_{2}(\alpha)$ & $\frac{5 \alpha^{2}+37 \alpha+68}{\alpha^{2}+7 \alpha+14}$ & $\frac{54}{11}+\frac{\alpha}{11}$ & $\frac{5 \alpha^{2}+37 \alpha+68}{\alpha^{2}+7 \alpha+14}$ \\
$\bar{x}_{2}(\alpha)$ & $\frac{7 \alpha^{2}+22 \alpha-139}{\alpha^{2}+3 \alpha-26}$ & $\frac{21}{4}-\frac{\alpha}{4}$ & $\frac{7 \alpha^{2}+22 \alpha-139}{\alpha^{2}+3 \alpha-26}$ \\
\hline
\end{tabular}


Example 2. In this example, let us consider $3 \times 3$ fully fuzzy system of linear equations with trapezoidal fuzzy numbers (Das \& Chakraverty 2012) as follows

$$
\begin{aligned}
& \left(\begin{array}{ccc}
(0.1,0.4,0.6,0.9) & (1,1.4,1.6,1.9) & (0.11,0.3,0.5,0.9) \\
(0.1,0.15,0.18,0.2) & (0.11,0.14,0.18,0.2) & (6,6.1,6.15,6.2) \\
(5,5.1,5.2,5.4) & (0.1,0.3,0.35,0.4) & (0.11,0.2,0.3,0.4)
\end{array}\right)\left(\begin{array}{c}
\tilde{x}_{1} \\
\tilde{x}_{2} \\
\tilde{x}_{3}
\end{array}\right) \\
& \quad=\left(\begin{array}{c}
(0.01,0.1,0.15,0.2) \\
(0.11,0.14,0.16,2) \\
(0.1,0.14,0.16,0.2)
\end{array}\right) .
\end{aligned}
$$

Now using the $\alpha$-cut approach, the above system can be reduced to

$$
\begin{gathered}
\left(\begin{array}{ccc}
{[0.3 \alpha+0.1,-0.3 \alpha+0.9]} & {[0.4 \alpha+1,-0.3 \alpha+1.9]} & {[0.19 \alpha+0.11,-0.4 \alpha+0.9]} \\
{[0.05 \alpha+0.1,-0.02 \alpha+0.2]} & {[0.03 \alpha+0.11,-0.02 \alpha+0.2]} & {[0.1 \alpha+6,-0.05 \alpha+6.2]} \\
{[0.1 \alpha+5,-0.2 \alpha+5.4]} & {[0.2 \alpha+0.1,-0.05 \alpha+0.4]} & {[0.09 \alpha+0.11,-0.1 \alpha+0.4]}
\end{array}\right) \\
\left(\begin{array}{c}
\tilde{x}_{1} \\
\tilde{x}_{2} \\
\tilde{x}_{3}
\end{array}\right)=\left(\begin{array}{c}
{[0.09 \alpha+0.01,-0.05 \alpha+0.2]} \\
{[0.03 \alpha+0.11,-0.04 \alpha+0.2]} \\
{[0.04 \alpha+0.1,-0.04 \alpha+0.2]}
\end{array}\right) .
\end{gathered}
$$

Finally, using the proposed methods one may have

$$
\begin{aligned}
\underline{x}_{1}(\alpha) & =\frac{125 \alpha^{3}+7993 \alpha^{2}-409017 \alpha+582021}{10\left(166 \alpha^{3}-5386 \alpha^{2}-1266749 \alpha-2987081\right)}, \\
\bar{x}_{1}(\alpha) & =\frac{-\left(29 \alpha^{3}+10856 \alpha^{2}-123600 \alpha+352000\right)}{10\left(21 \alpha^{3}-6281 \alpha^{2}-226540 \alpha-1208000\right)}, \\
\underline{x}_{2}(\alpha) & =\frac{17 \alpha^{3}+482 \alpha^{2}-478099 \alpha-36340}{2\left(166 \alpha^{3}-5386 \alpha^{2}-1266749 \alpha-2987081\right)} \\
\bar{x}_{2}(\alpha) & =\frac{-2\left(94 \alpha^{3}-4659 \alpha^{2}-39150 \alpha+235000\right)}{5\left(21 \alpha^{3}-6281 \alpha^{2}-226540 \alpha-1208000\right)} \\
\underline{x}_{3}(\alpha) & =\frac{41 \alpha^{3}-4387 \alpha^{2}-31628 \alpha-53460}{166 \alpha^{3}-5386 \alpha^{2}-1266749 \alpha-2987081},
\end{aligned}
$$

and

$$
\bar{x}_{3}(\alpha)=\frac{33 \alpha^{3}-1490 \alpha^{2}+13500 \alpha-34800}{21 \alpha^{3}-6281 \alpha^{2}-226540 \alpha-1208000} .
$$

The solutions obtained by the proposed methods are exactly same as Das \& Chakraverty (2012).

Example 3. Let us assume that the omega manufacturing (Senthilkumar \& Rajendran 2011; Ezzati et al 2012) company has decided to produce three products, namely Product 1, Product 2 and Product 3 . The available capacity of the machines that might limit output is summarized (table 2). 
Table 2. Available capacity of the machines.

\begin{tabular}{lc}
\hline Machine type & Available time (machine hours per month) \\
\hline Milling & $(19,68,115)$ \\
Lathe & $(30,77,261)$ \\
Grinder & $(61,167,253)$ \\
\hline
\end{tabular}

Table 3. Product coefficient (in machines hours per unit).

\begin{tabular}{lccr}
\hline Machine type & Product 1 & Product 2 & Product 3 \\
\hline Milling & $(1,2,5)$ & $(3,4,4)$ & $(0,1,2)$ \\
Lathe & $(2,3,5)$ & $(0,1,11)$ & $(4,5,6)$ \\
Grinder & $(2,5,7)$ & $(4,6,6)$ & $(5,7,10)$ \\
\hline
\end{tabular}

The number of machine hours required for each unit of the respective product is given below (table 3):

Now, we have to determine how much of each product should be produced to utilize the entire available time.

To model the above problem, we may represent $\tilde{x}_{1}, \tilde{x}_{2}$ and $\tilde{x}_{3}$ as the quantity of the products 1,2 and 3 , respectively produced during the month. Corresponding fully fuzzy linear system for the above problem may then be written as

$$
\left(\begin{array}{ccc}
(1,2,5) & (3,4,4) & (0,1,2) \\
(2,3,5) & (0,1,11) & (4,5,6) \\
(2,5,7) & (4,6,6) & (5,7,10)
\end{array}\right)\left(\begin{array}{c}
\tilde{x}_{1} \\
\tilde{x}_{2} \\
\tilde{x}_{3}
\end{array}\right)=\left(\begin{array}{c}
(19,68,115) \\
(30,77,261) \\
(61,167,253)
\end{array}\right)
$$

Using the proposed methods, we obtain the solution as

$$
\left(\begin{array}{c}
\tilde{x}_{1} \\
\tilde{x}_{2} \\
\tilde{x}_{3}
\end{array}\right)=\left(\begin{array}{c}
(1,5,7) \\
(6,12,14) \\
(7,10,12)
\end{array}\right)
$$

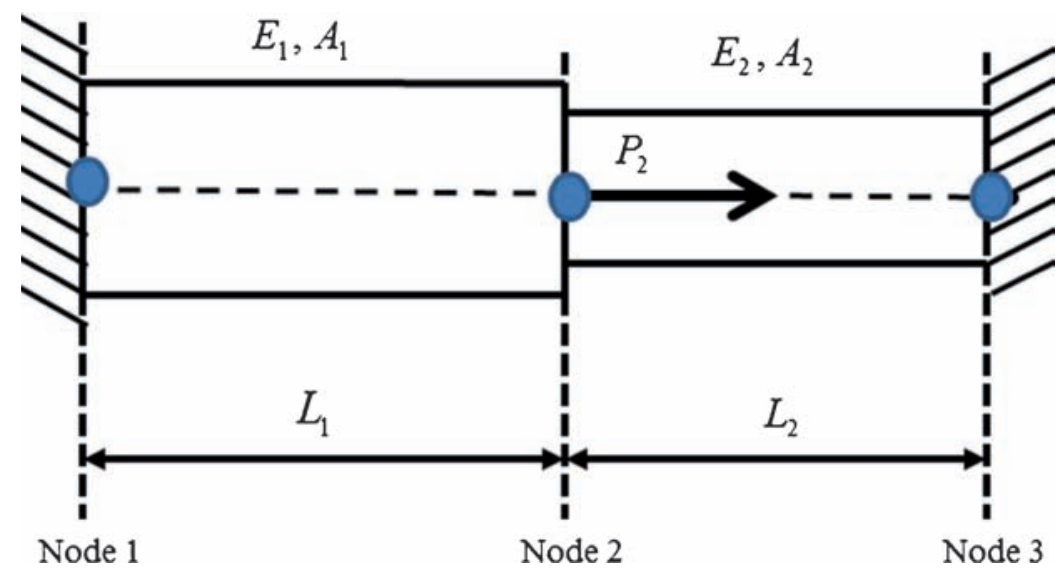

Figure 2. Fixed-fixed rectangular bar. 
Table 4. Data for two-stepped bar (Example 4).

\begin{tabular}{lcccc}
\hline Parameters & Case A & Case B & Case C & Case D \\
\hline$A_{1}\left(\mathrm{~mm}^{2}\right)$ & 2400 & 2400 & $(2390,2400,2410)$ & $(2390,2400,2410)$ \\
$A_{2}\left(\mathrm{~mm}^{2}\right)$ & 1200 & 1200 & $(1195,1200,1205)$ & $(1195,1200,1205)$ \\
$L_{1}(\mathrm{~mm})$ & 300 & 300 & 300 & 300 \\
$L_{2}(\mathrm{~mm})$ & 400 & 400 & 400 & 400 \\
$E_{1}\left(\mathrm{~N} / \mathrm{mm}^{2}\right)$ & $0.7 \times 10^{5}$ & $(0.69,0.7,0.71) \times 10^{5}$ & $0.7 \times 10^{5}$ & $(0.69,0.7,0.71) \times 10^{5}$ \\
$E_{2}\left(\mathrm{~N} / \mathrm{mm}^{2}\right)$ & $2 \times 10^{5}$ & $(1.95,2,2.05) \times 10^{5}$ & $2 \times 10^{5}$ & $(1.95,2,2.05) \times 10^{5}$ \\
$P_{2}(\mathrm{~N})$ & $400 \times 10^{3}$ & $(375,400,425) \times 10^{3}$ & $(375,400,425) \times 10^{3}$ & $(375,400,425) \times 10^{3}$ \\
\hline
\end{tabular}

This problem is also solved by the method of Ezzati et al (2012) and found that the present results are in good agreement.

Example 4. In this example, we have considered a two-step fixed-fixed rectangular bar as shown in figure 2. For the uncertain static response of structure at node 2, input variables are shown in table 4. To obtain the static response, here fuzzy finite element method is used with the proposed methodologies. We know that (Behera and Chakraverty 2013a, c) for static analysis fuzzy finite element method converts the problem to a fuzzy or fully fuzzy system of linear equations. Accordingly, with fuzzy inputs the corresponding problem converts to a fully fuzzy system of linear equations. Here, four different Cases A, B, C and D have been considered. For Case A, cross sectional area $\left(A_{i}\right)$, Length $\left(L_{i}\right)$, Young's modules $\left(E_{i}\right)$ and applied force $\left(P_{2}\right)$ are considered as deterministic in nature. But, Young's modules $\left(E_{i}\right)$ and applied force $\left(P_{2}\right)$ are assumed as triangular fuzzy number for Case B. In Case $\mathrm{C}$, cross sectional area $\left(A_{i}\right)$ and applied force $\left(P_{2}\right)$ are taken as uncertain. Lastly, for Case D Young's modules $\left(E_{i}\right)$, cross sectional area $\left(A_{i}\right)$ and applied force $\left(P_{2}\right)$ are considered as fuzzy number.

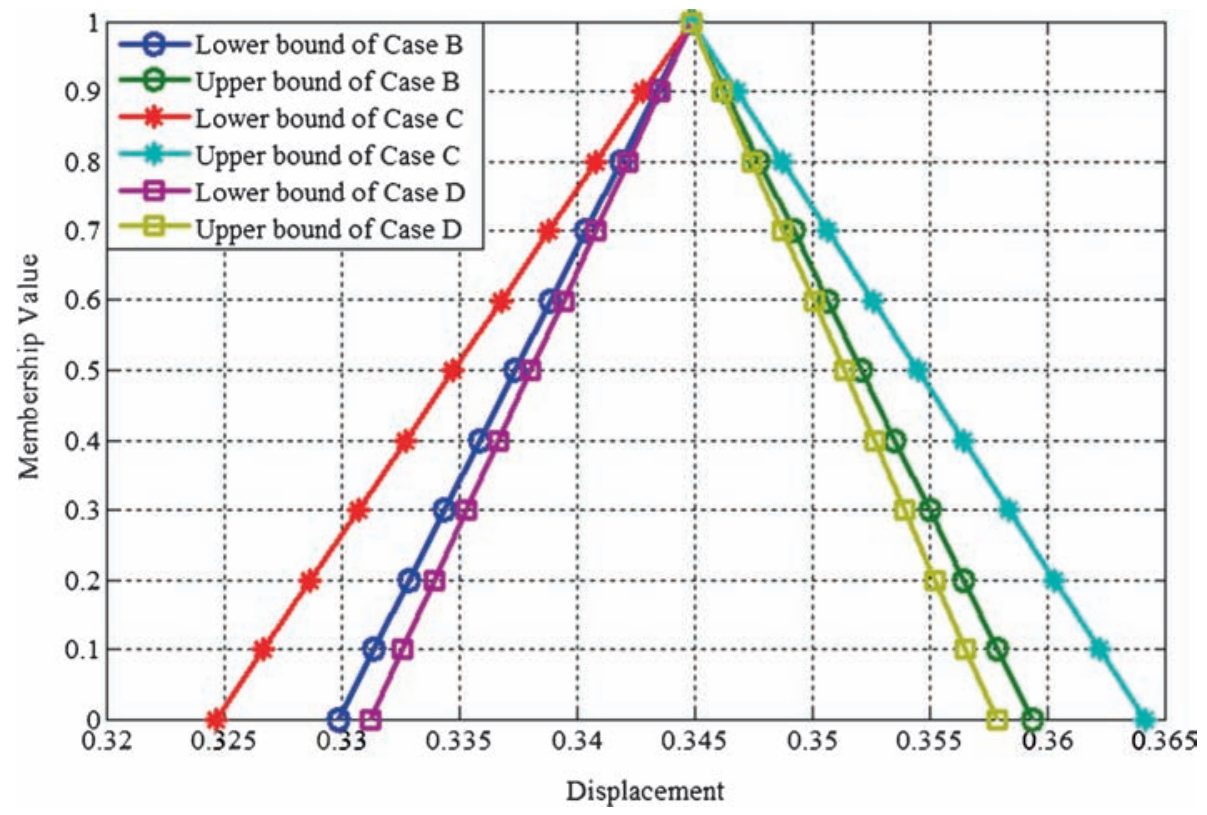

Figure 3. Displacement at node 2 for different cases (Example 4). 
Using traditional finite element method for Case A, one may obtain the static displacement at node 2 as $0.3448 \mathrm{~mm}$. For Cases B, C and D, fuzzy finite element method is applied with the proposed methodologies to compute the fuzzy nodal displacements respectively as $(0.3298$, $0.3448,0.3593),(0.3246,0.3448,0.3641)$ and $(0.3312,0.3448,0.3578)$. Obtained results for Cases B, C and D are also depicted in figure 3. From the results, it may be noted that uncertainty width is maximum for Case $\mathrm{C}$ and minimum for Case $\mathrm{D}$. For this problem area of cross sections are more sensitive than Young's modules. It is worth mentioning that the centre values of Cases $\mathrm{B}, \mathrm{C}$ and D exactly coincide with Case A.

\section{Conclusions}

In this paper, non-negative solution of fully fuzzy generalized system of linear equations is considered. Related to this, two new methods are proposed based on single and double parametric form of the fuzzy numbers. The double parametric approach found to be easy and straight forward. This method keeps the order of original system unchanged. Triangular and trapezoidal fuzzy numbers have been considered for the analysis. Obtained results are compared with the existing results to show the efficiency and powerfulness of the methodologies.

\section{Acknowledgements}

This work was financially supported by the Board of Research in Nuclear Sciences (Department of Atomic Energy), Government of India. We would also like to thank the anonymous referees and the editor for various valuable comments and suggestions to improve the quality of the paper.

\section{References}

Abbasbandy S and Jafarian A 2006 Steepest descent method for system of fuzzy linear equations. Appl. Math. Comput. 175: 823-833

Abbasbandy S, Jafarian A and Ezzati R 2005 Conjugate gradient method for fuzzy symmetric positivedefinite system of linear equations. Appl. Math. Comput. 171: 1184-1191

Allahviranloo T 2004 Successive overrelaxation iterative method for fuzzy system of linear equations. Appl. Math. Comput. 162: 189-196

Allahviranloo T 2005 The adomian decomposition method for fuzzy system of linear equations. Appl. Math. Comp. 163: 553-563

Allahviranloo T and Mikaeilvand N 2011a Fully fuzzy linear systems solving using MOLP. World Appl. Sci. J. 12: 2268-2273

Allahviranloo T and Mikaeilvand N 2011b Non zero solutions of the fully fuzzy linear systems. Appl. Comput. Math. 10: 271-282

Babbar N, Kumar A and Bansal A 2013 Solving fully fuzzy linear system with arbitrary triangular fuzzy numbers $(m, \alpha, \beta)$. Soft Comput. 17: 691-702

Behera D and Chakraverty S 2012 A new method for solving real and complex fuzzy system of linear equations. Comput. Math. Model. 23: 507-518

Behera D and Chakraverty S 2013a Fuzzy analysis of structures with imprecisely defined properties. Comput. Model. Eng. Sci. 96: 317-337

Behera D and Chakraverty S 2013b Fuzzy centre based solution of fuzzy complex linear system of equations. Internat. J. Uncertain. Fuzziness Knowledge-Based Systems 21: 629-642

Behera D and Chakraverty S 2013c Fuzzy finite element analysis of imprecisely defined structures with fuzzy nodal force. Eng. Appl. Artificial Intel. 26: 2458-2466 
Behera D and Chakraverty S 2013d Fuzzy Finite Element based solution of uncertain static problems of structural mechanics. Int. J. Comput. Appl. 69: 6-11

Behera D and Chakraverty S 2013e Solution method for fuzzy system of linear equations with crisp coefficients. Fuzzy Inf. Eng. 5: 205-219

Behera D and Chakraverty S 2014 Solving fuzzy complex system of linear equations. Inform. Sci. 277: $154-162$

Chakraverty S and Behera D 2013 Fuzzy system of linear equations with crisp coefficients. J. Int. Fuzzy Syst. 25: 201-207

Das S and Chakraverty S 2012 Numerical solution of interval and fuzzy system of linear equations. Appl. Appl. Math.: Int. J. (AAM) 7: 334-356

Dehghan M and Hashemi B 2006 Solution of the fully fuzzy linear system using the decomposition procedure. Appl. Math. Comput. 182: 1568-1580

Dehghan M, Hashemi B and Ghatee M 2006 Computational methods for solving fully fuzzy linear systems. Appl. Math. Comput. 179: 328-343

Dehghan M, Hashemi B and Ghatee M 2007 Solution of the fully fuzzy linear system using iterative techniques. Chaos Soli. Fract. 34: 316-336

DeMarr R 1972 Nonnegative matrices with nonnegative inverses. Proc. Am. Mathematical Soc. 35: 307308

Dubois D and Prade H 1980 Fuzzy sets and systems: Theory and applications. New York: Academic Press

Ezzati R, Khezerloo S and Yousefzadeh A 2012 Solving fully fuzzy linear system of equations in general form, Journal of Fuzzy Set Valued Analysis, DOI:10.5899/2012/jfsva-00117

Friedman M, Ming M and Kandel A 1998 Fuzzy linear systems. Fuzzy Sets Syst. 96: 201-209

Hanss M 2005 Applied fuzzy arithmetic: An introduction with engineering applications. Berlin: SpringerVerlag

Kaufmann A and Gupta M M 1988 Fuzzy mathematical models in engineering and management science. Amsterdam: North Holland Publishers

Kumar A, Bansal A and Neetu 2011 Solution of fully fuzzy linear system with arbitrary coefficients. Int. J. Appl. Math. Comput. 3: 232-237

Mosleh M, Otadi M and Khanmirzaie A 2009 Decomposition method for solving fully fuzzy linear systems. Iranian J. Optimis. 1: 188-198

Muzzioli S and Reynaerts H 2006 Fuzzy linear systems of the form $A_{1} x+b_{1}=A_{2} x+b_{2}$. Fuzzy Sets Syst. 157: 939-951

Muzzioli S and Reynaerts H 2007 The solution of fuzzy linear systems by non-linear programming: a financial application. European J. Oper. Res. 177: 1218-1231

Nasseri S H, Sohrabi M and Ardil E 2008 Solving fully fuzzy linear systems by use of a certain decomposition of the coefficient matrix. Int. J. Comput. Math. Sci. 2: 140-142

Otadi M and Mosleh M 2012 Solving fully fuzzy matrix equations. Appl. Math. Model. 36: 6114-6121

Ross T J 2004 Fuzzy logic with engineering applications. New York: John Wiley \& Sons

Senthilkumar P and Rajendran G 2011 New approach to solve symmetric fully fuzzy linear systems. Sadhana 36: 933-940

Sun X and Guo S 2009 Solution to general fuzzy linear system and its necessary and sufficient condition. Fuzzy Inf. Eng. 3: 317-327

Vijayalakshmi V 2011 ST decomposition method for solving fully fuzzy linear systems using Gauss Jordan for trapezoidal fuzzy matrices. Int. Math. Forum 6: 2245-2254

Yin J F and Wang K 2009 Splitting iterative methods for fuzzy system of linear equations. Comput. Math. Model. 20: 326-335

Zadeh L A 1965 Fuzzy sets, Inf. Control 8: 338-353

Zadeh L A, Fu K S and Shimura M 1975 Fuzzy sets and their applications to cognitive and decision processes. New York/San Francisco/London: Academic Press

Zimmermann H J 2001 Fuzzy set theory and its application. Boston/Dordrecht/London: Kluwer Academic Publishers 